\title{
Bayesianism and Irrelevant Conjunction*
}

\author{
Patrick M aherł‡
}

Bayesian confirmation theory offers an explicatum for a pretheoretic concept of confirmation. The "problem of irrelevant conjunction" for this theory is that, according to some people's intuitions, the pretheoretic concept differs from the explicatum with regard to conjunctions involving irrelevant propositions. Previous Bayesian solutions to this problem consist in showing that irrelevant conjuncts reduce the degree of confirmation; they have the drawbacks that (i) they don't hold for all ways of measuring degree of confirmation and (ii) they don't remove the conflict with intuition but merely "soften the impact" (as Fitelson has written). A better solution, which avoids both these drawbacks, is to show that the intuition is wrong.

1. Bayesian C onfirmation Theory. Philosophers are often concerned with finding a precise replacement for a vague concept of ordinary language. The vague concept is then called the explicandum and a proposed precise replacement for it is called the explicatum; the activity of proposing an explicatum is called explication. Since the explicandum is vague and the explicatum is precise, these two concepts are not identical; what is required for a good explicatum is that it be similar to the explicandum and theoretically fruitful and simple. (See Carnap 1950, ch. 1 for further discussion.)

Bayesianism is best conceived as offering an explicatum for a vague concept of inductive probability that is used in ordinary language. Clarification of the nature of this explicandum is an important topic but will not be pursued here. I will simply remark that inductive probabilities often do not have numeric values. The Bayesian explicatum for inductive probability is a real-valued function $p$ satisfying the laws of mathematical

*R eceived N ovember 2003; revised M arch 2004

†To contact the author write to $105 \mathrm{G}$ regory H all, 810 South Wright Street, U rbana, IL 61801; e-mail: patrick@maher1.net.

$\ddagger$ thank Branden Fitelson and the referees for their comments on earlier versions of this paper.

Philosophy of Science, 71 (October 2004) pp. 515-520. 0031-8248/ 2004/ 7104-0006 $\$ 10.00$ Copyright 2004 by the Philosophy of Science Association. All rights reserved. 
probability. I will here assume that $p$ is a conditional probability function, with $p(H \mid E)$ being the explicatum for the inductive probability of $H$ given E.

Bayesian confirmation theory offers an explicatum for a vague concept which I will for now simply refer to as "confirmation". O ne of the main things that needs to be done in this essay is to clarify what this explicandum is, but that will be more easily done after I have laid out the Bayesian explicatum and the problem of irrelevant conjunction. I will therefore postpone the clarification of the explicandum until Section 4; in the meantime, I will use 'confirm' and its cognates to designate whatever concept is the explicandum for Bayesian confirmation theory.

Let us define a predicate $C$ as follows:

Definition 1. $C(H, E, K)$ iff $p(H \mid E . K)>p(H \mid K)$.

Then the Bayesian explicatum for " $E$ confirms $H$ given $K$ " is $C(H, E$, K).

2. The Problem of Irrelevant C onjunction. It is easy to prove:

Theorem 1. If $H . K$ entails $E$ then $C(H . X, E, K)$ provided that $p(H . X \mid K)>0$ and $0<p(E \mid K)<1$.

It follows that $\mathrm{C}(\mathrm{H} . \mathrm{X}, \mathrm{E}, \mathrm{K})$ can be true in cases in which $\mathrm{X}$ is intuitively irrelevant to $\mathrm{H}$ and $\mathrm{E}$. Earman $(1992,64 f$.) and R osenkrantz (1994, 470f.) are Bayesians who seem to think that this theorem is a problem that requires some kind of solution. Fitelson (2002) agrees that there is a problem but argues that the problem is not Theorem 1 but rather: ${ }^{1}$

Theorem 2. If $C(H, E, K)$ then $C(H . X, E, K)$ provided that $p(H . X \mid K)>0, \quad 0<p(E \mid K)<1, \quad p(H . X \mid K)=p(H \mid K) p(X \mid K)$, $p(E . X \mid K)=p(E \mid K) p(X \mid K)$, and $p(H . E . X \mid K)=p(H . E \mid K) p(X \mid K)$.

Theorem 2 also implies that $\mathrm{C}(\mathrm{H} . \mathrm{X}, \mathrm{E}, \mathrm{K})$ can be true in cases in which $X$ is intuitively irrelevant to $H$ and $E$ but, unlike Theorem 1, Theorem 2 is not restricted to the case where $\mathrm{H}$.K entails $\mathrm{E}$. $\mathrm{H}$ awthorne and $\mathrm{F}$ itelson (2004) prove a stronger version of Theorem 2.

Why is either of these theorems a "problem?" The definition of $C$ was stipulative, so there can be no question about its correctness. Theorems 1 and 2 demonstrably follow from that definition, so there can be no question about their correctness either. Therefore, the "problem" can only be that the theorems seem to show that the explicatum $C$ differs from its

1. What follows is Theorem 1 of F itelson 2002, restated in my notation and including all the needed provisos. 
explicandum. The thought seems to be that the following appears intuitively to be true:

* If $X$ is irrelevant to $H$ and $E$ (given $K$ ) then $E$ does not confirm $\mathrm{H}$.X (given K).

Since the analog of $*$ for $C$ is not true (as Theorems 1 and 2 show), it follows that $C$ is in this respect not a good explicatum for confirmation. That is the problem.

3. Previous Solutions. Earman, R osenkrantz, and F itelson all offer Bayesian solutions to the problem of irrelevant conjunction. A lthough they differ in detail, they all have the same general idea, which is to show that when $\mathrm{X}$ is irrelevant then $\mathrm{H} . \mathrm{X}$ is confirmed to a lesser degree than $\mathrm{H}$ is.

In order to show this they must make some assumptions about how to measure degree of confirmation. The most popular Bayesian measures of the degree to which $\mathrm{E}$ confirms $\mathrm{H}$ given $\mathrm{K}$ are the following.

$$
\begin{array}{lll}
\text { Difference measure: } & d(H, E, K)=p(H \mid E . K)-p(H \mid K), \\
\text { Ratio measure: } & r(H, E, K)=p(H \mid E . K) / p(H \mid K), \\
\text { Likelihood ratio: } & \mid(H, E, K)=p(E \mid H . K) / p(E \mid \sim H . K) .
\end{array}
$$

Earman and R osenkrantz assumed that degree of confirmation is measured by $d$; only Fitelson took account of the fact that there are other possible measures. Fitelson's solution is to show that if the conditions of Theorem 2 hold and $p(X \mid K)<1$ then $d(H . X, E, K)<d(H, E, K)$ and $l(H . X, E, K)<l(H, E, K)$. F itelson notes that under the same conditions $r(H . X, E, K)=r(H, E, K)$ and so the solution fails if degree of confirmation is measured by $r$. Fitelson deals with this by (a) observing that Earman's and R osenkrantz's solutions also fail if $r$ is used and (b) arguing that $r$ is not a good measure of degree of confirmation ( $F$ itelson 2002, 618).

The solutions of these authors have two drawbacks. F irst, as just noted, they all fail if degree of confirmation is measured by $r$, so advocates of these solutions need to argue that $r$ is not a good measure of degree of confirmation. Since $r$ (or its logarithm) has had some important advocates (e.g., H orwich 1982; M ilne 1996; $K$ uipers 2000), there is a significant issue here and it would be preferable to have a solution to the problem of irrelevant conjunction that does not depend on ruling out $r$.

The second drawback of the solutions offered by these authors is that they do not fully solve the problem. R ecall that the problem arose because * has seemed intuitively to be true, from which it follows that $C(H, E$, $\mathrm{K}$ ) is true in many cases in which $\mathrm{E}$ does not confirm $\mathrm{H}$ given $\mathrm{K}$, and hence that $C$ is in this respect not a good explicatum for confirmation. 
The solutions offered by our authors do not deny any of this, which is why I say that they do not fully solve the problem. What these solutions attempt to do is merely to "soften the impact" (Fitelson 2002, 611); a solution that removed the impact altogether would be better.

4. Clarification of the Explicandum. So far I have been using the term 'confirm' and its cognates to designate the explicandum for Bayesian confirmation theory, whatever that may be. But we can't properly assess the problem of irrelevant conjunction, or indeed Bayesian confirmation theory, without knowing what this explicandum is. I will now address this issue.

Let us adopt the following stipulative definition:

D efinition 2. $\mathrm{E}$-confirms $\mathrm{H}$ given $\mathrm{K}$ iff the inductive probability of $H$ given $E$ and $K$ is greater than the inductive probability of $H$ given $\mathrm{K}$ alone.

Since inductive probability is a vague concept of ordinary language, so is $r$-confirmation.

There are some good reasons to take the explicandum for Bayesian confirmation theory to be $r$-confirmation. One is that if $p$ is a good explicatum for inductive probability, as Bayesianism maintains, then $\mathrm{C}$ must be a good explicatum for $\mathrm{r}$-confirmation, and in fact $\mathrm{C}$ seems tailor-made to explicate r-confirmation.

A nother consideration is that the term 'confirmation' has sometimes been used in the literature of confirmation theory to mean r-confirmation. F or example, $\mathrm{N}$ icod $(1923,1970,189)$ stipulated that he would use 'confirmation' to mean r-confirmation. A lso Carnap's (1950) attempts to clarify what he called the "classificatory concept of confirmation" show that he had in mind r-confirmation.

A $n$ alternative possibility is to take the explicandum to be the concept that ordinary people and scientists mean when they talk of "confirmation." But if this ordinary concept is $r$-confirmation, then this suggestion is equivalent to the preceding one. On the other hand, if the ordinary concept of confirmation differs from $\mathrm{r}$-confirmation, then $\mathrm{C}$ will be a poor explicatum for it.

To illustrate this last point, suppose it is true (as I think it is) that in ordinary language, including science, $\mathrm{E}$ confirms $\mathrm{H}$ given $\mathrm{K}$ only if $\mathrm{H}$ has a high inductive probability given $E$ and $K$. Since $C(H, E, K)$ is true in many cases in which $p(H \mid E . K)$ is small, it follows that if $p$ is a good explicatum for inductive probability then $\mathrm{C}(\mathrm{H}, \mathrm{E}, \mathrm{K})$ is true in many cases in which $\mathrm{H}$ is very improbable, and hence the explicatum $\mathrm{C}$ diverges fundamentally from its explicandum.

A nother example: Goodman (1979, 68f.), G emes (1994, 598f.), and 
others apparently believe that in ordinary language, $\mathrm{E}$ confirms $\mathrm{H}$ if and only if $E$ raises the inductive probability of every "component" or "part" of $\mathrm{H}$. I don't myself think that this condition is either necessary or sufficient. But if these philosophers were right, then $\mathrm{C}$ would again be a very poor explicatum for the ordinary language concept of confirmation. F or example, Carnap $(1950,395)$ showed that it is possible to have:

$$
\mathrm{C}\left(\mathrm{H}_{1} . \mathrm{H}_{2}, \mathrm{E}, \mathrm{K}\right) . \sim \mathrm{C}\left(\mathrm{H}_{1}, \mathrm{E}, \mathrm{K}\right) . \sim \mathrm{C}\left(\mathrm{H}_{2}, \mathrm{E}, \mathrm{K}\right) \text {. }
$$

If $\mathrm{H}_{1}$ and $\mathrm{H}_{2}$ are components or parts of $\mathrm{H}_{1} \cdot \mathrm{H}_{2}$ then this result would show a serious discrepancy between $\mathrm{C}$ and the ordinary concept, if $\mathrm{G}$ oodman et al. are right about the ordinary concept.

In short, Bayesian confirmation theorists must choose between these options:

i. The explicandum for Bayesian confirmation theory is r-confirmation. In that case, $C$ is a good explicatum provided $p$ is.

ii. The explicandum differs from r-confirmation. Then $C$ is not a good explicatum even if $p$ is.

It seems clear to me that the best choice for Bayesian confirmation theorists is i. I will assume this in what follows. If there are Bayesian confirmation theorists who disagree with this choice, then they owe us an account of what exactly their explicandum is and why they persist with $\mathrm{C}$ as their explicatum.

5. A B etter Solution. N ow that we have decided what concept Bayesian confirmation theory is trying to explicate, we can solve the problem of irrelevant conjunction. If this problem is not just an equivocation, then the term 'confirms' in * must mean 'r-confirms.' But then it is easy to see that $*$ is false. For example, if $X$ is not certainly false and $H$ is not certainly true and $\mathrm{X}$ is irrelevant to $\mathrm{H}$, then $\mathrm{H}$ raises the inductive probability of H.X, and hence it r-confirms H.X, contrary to $*$.

$H$ ere is a numerical example: Suppose $K$ tells us that a coin is fair, let $\mathrm{H}$ be that the coin lands heads on the first toss, and let $\mathrm{X}$ be that it lands heads on the second toss. Thus $X$ is irrelevant to $H$ given $K$. This is a case where inductive probabilities have precise quantitative values; the inductive probability of $H . X$ is $1 / 4$ given just $K$ and is $1 / 2$ given $H$ and $\mathrm{K}$, so $\mathrm{H} \mathrm{r}$-confirms $\mathrm{H} . \mathrm{X}$ given $\mathrm{K}$, contrary to $*$.

The observation that $*$ is false solves the problem of irrelevant conjunction without either of the drawbacks of previous Bayesian solutions. It does not depend on a choice of measure of degree of confirmation and it fully solves the problem. The reason Bayesian confirmation theorists have not seen this solution before is that they have not been clear about what their explicandum is. 


\section{REFERENCES}

Carnap, R udolf (1950), L ogical F oundations of P robability, 2d ed., 1962. Chicago: U niversity of Chicago Press.

Earman, John (1992), Bayes or Bust? A Critical Examination of Bayesian Confirmation Theory. Cambridge, M A : M IT Press.

Fitelson, Branden (2002), "Putting the Irrelevance Back into the Problem of Irrelevant Conjunction", Philosophy of Science 69: 611-622.

Gemes, K en (1994), "A N ew Theory of Content I: Basic Content", J ournal of P hilosophical Logic 23: 595-620.

Goodman, N elson (1979), F act, Fiction, and Forecast, 3d ed. Indianapolis: H ackett.

$\mathrm{H}$ awthorne, James, and Branden Fitelson (2004), "R e-solving Irrelevant Conjunction with Probabilistic Independence", P hilosophy of Science 71: 505-514.

H orwich, Paul (1982), Probability and Evidence. Cambridge: Cambridge U niversity Press.

K uipers, Theo A. F. (2000), From Instrumentalism to Constructive Realism. D ordrecht: K luwer.

M ilne, Peter (1996), "Log[p(h/eb)/p(h/b)] is the One True M easure of Confirmation", Philosophy of Science 63: 21-26.

$\mathrm{N}$ icod, J ean (1923), L e P roblème L ogique de I'Induction. Paris: A Ican.

- - - (1970), Geometry and Induction. [English translation of works originally published in F rench in 1923 and 1924.] Berkeley and L os A ngeles: U niversity of California Press. R osenkrantz, R. D. (1994), "Bayesian Confirmation: Paradise R egained", B ritish J ournal for the Philosophy of Science 45: 467-476. 